\title{
WHOOPING COUGH ENCEPHALITIS
}

\author{
BY \\ A. L. WOOLF and H. CAPLIN \\ From the Midland Centre for Neurosurgery, Smethwick, and the Connaught Hospital, London
}

(RECEIVED FOR PUBLICATION JANUARY 2, 1956)

It is the purpose of this paper to report a case in which fatal convulsions occurred during convalescence from whooping cough, at a time when paroxysms of coughing were absent. This case is not unique, a similar one having been reported by Hiller and Grinker (1930). These authors point out that while air embolism or circulatory stasis may be a satisfactory explanation of convulsions associated with severe paroxysms of coughing, some other mechanism must be responsible for convulsions occurring apart from such paroxysms. A study of the case reported below suggested an alternative explanation.

\section{Case Report}

The patient, a girl aged 3 years, was admitted to the Connaught Hospital on September 3, 1951. The child was born by a normal full-term delivery without any jaundice. She had been quite well until this infection with whooping cough. She had been inoculated with diphtheria toxoid at 18 months, but had not received any of the pertussis vaccines being used at the time.

There is no history of fits on either the maternal or paternal sides of the family, the other two children, both boys, being quite well and also free from fits.

Six to seven weeks before admission, the child developed a cough which was diagnosed as whooping cough with mild paroxysms (these were never very severe). Two weeks before admission, the mother noticed that the child was a little more fractious than she had been previously, with a slightly puffy face and with slightly swollen glands in the neck. The puffiness and swelling had disappeared two days later. The day before admission the child seemed very well and was helping her father in the garden and had no paroxysms of cough, although an occasional isolated cough was heard. She was put to bed and although the parents heard an occasional cough during the night, the mother is quite certain that there were no paroxysms. The following morning the child vomited twice and was listless and drowsy, lying curled up in a chair with no appetite. On the afternoon of admission, she appeared dazed and drowsy and would not respond to questions. On the way to hospital in an ambulance, she started to have a fit which lasted for about 15 minutes. She was given phenobarbitone, grain 1, and the convulsions ceased, but she remained stuporose.

On examination, she was dazed. The head and eyes were turned to the left side, there were no convulsions, but the arms were held stiffly and twitched slightly. The tendon reflexes were present and equal, but the abdominal reflexes were absent. The right plantar response was not obtained and the left was flexor.

At 11.25 p.m. she started to have a fit. The fit started on the left side of the face and the left arm and later became generalized. She was given soluble phenobarbitone, grain 1, intramuscularly. At 11.50 p.m. the fit was still continuing in the face on both sides and in both arms though it was less severe in the legs. She was given paraldehyde, $2 \mathrm{ml}$., intramuscularly.

On September 4 at 1 a.m. she was still having convulsions, but they were now entirely confined to the eyes, left arm and left leg, and they were not as violent as before. Her respirations were rather irregular and shallow. Her pulse was very rapid. At 7.30 a.m. she had another fit, but this time it was entirely right sided.

At 6 p.m. on the same day, she seemed drowsy and lay curled up. She did not cry when moved, but curled up again. She did not respond when her name was called but the ward sister said that she spoke rationally once or twice during the day. The abdominal reflexes were now present but sluggish. The knee and ankle jerks were present and equal but the knee jerks were sluggish. The plantar responses were both flexor. A lumbar puncture was performed, which yielded clear C.S.F. under a pressure of $230 \mathrm{~mm}$. Crystalline penicillin, 100,000 units, were given intrathecally. At 8.30 p.m. the child was more alert and was talking rationally. On September 5, she was dehydrated and stuporose, she had a left hemiplegia and could not close the left eye. The left arm and leg were spastic and the abdominal reflexes were absent. The knee and ankle jerks were present and equal. The right plantar response was not obtained and the left was extensor. Pin-prick was appreciated in the left foot and leg, but she did not withdraw the leg. The C.S.F. was under a pressure of $220 \mathrm{~mm}$., there were 80 lymphocytes per c.mm. and $50 \mathrm{mg}$. of glucose per $100 \mathrm{ml}$.

She was given a gastric drip and put in an oxygen tent; chloramphenicol, $0.5 \mathrm{~g}$., was given six hourly and the penicillin, 100,000 units, was continued six-hourly intramuscularly. 
By the afternoon, she seemed rather better, her colour was improved and her breathing was quieter. She moved the left arm and leg when pricked. At 5.30 p.m. she suddenly went blue and the right arm became rigid. Her heart continued to beat for about 15 minutes, but in spite of artificial respiration she died.

Necropsy. The cardiovascular system showed no abnormality apart from a few petechial haemorrhages on the epicardium.

The trachea and bronchi contained a little viscid mucus.

The lungs showed small patches of collapse at the bases with sub-pleural petechiae. The mediastinal lymph nodes were slightly enlarged. The liver and spleen were congested, the spleen being slightly larger than normal.

The mesenteric and pre-aortic lymph nodes were slightly enlarged. The stomach and intestines were normal. The pancreas was congested. The kidneys appeared normal. The thyroid and suprarenal glands were normal.

Histology of the spleen revealed congestion of the pulp. The lymph nodes showed chronic inflammatory reactive changes.

The brain was fixed in formalin and then examined. It weighed $1,555 \mathrm{~g}$. and was unusually pale. It showed great swelling of the cerebral convolutions and the cerebral hemispheres were softer than normal.

On cutting through the brain, the white matter of both hemispheres was extremely soft, especially at the level of the thalamus. The softening reached its maximum on the right side at the level of the posterior horn of the lateral ventricle. In this region, the white matter was peppered with dilated, small blood vessels. Many of these vessels were surrounded by an indistinct, brownish halo less than $0.05 \mathrm{~cm}$. in diameter. In addition to the brownish haloes, there was a general discoloration, mainly occupying the centrum semi-ovale, and not extending intto the digitate white matter. The grey matter in the depth of the sulcus between the right inferior frontal convolution and the orbital gyrus appeared to be softened.

Histology. Throughout the cerebral hemisphere there was cuffing of blood vessels with lymphocytes, up to 10 layers thick. The cuffing was most prominent in the cerebral cortex of all areas, was much less marked in the white matter and was practically absent from the cerebellum. The cuffing was very prominent in all parts of the thalamus (Fig. 1 and 2) and the zona incerta, but much less obvious in the lentiform and caudate nuclei. There were occasional heavily cuffed vessels in the ventral part of the internal capsule, especially anteriorly. In the mid-brain, cuffing was marked around vessels in the superior colliculus and in the substantia nigra; in the pons, the periaqueductal grey matter was most involved (Fig. 3), but a few vessels in the floor of the fourth ventricle were cuffed as were also perforating vessels deep within the brain stem.

Frequently the lymphocytes extended beyond the perivascular space into the surrounding tissue, which

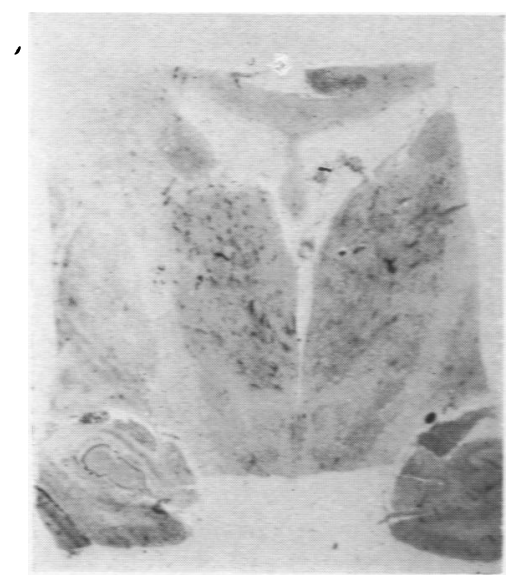

Fig. 1.-Thalamus, showing greatly increased prominence of vessels. The white matter is little affected. Nissl.

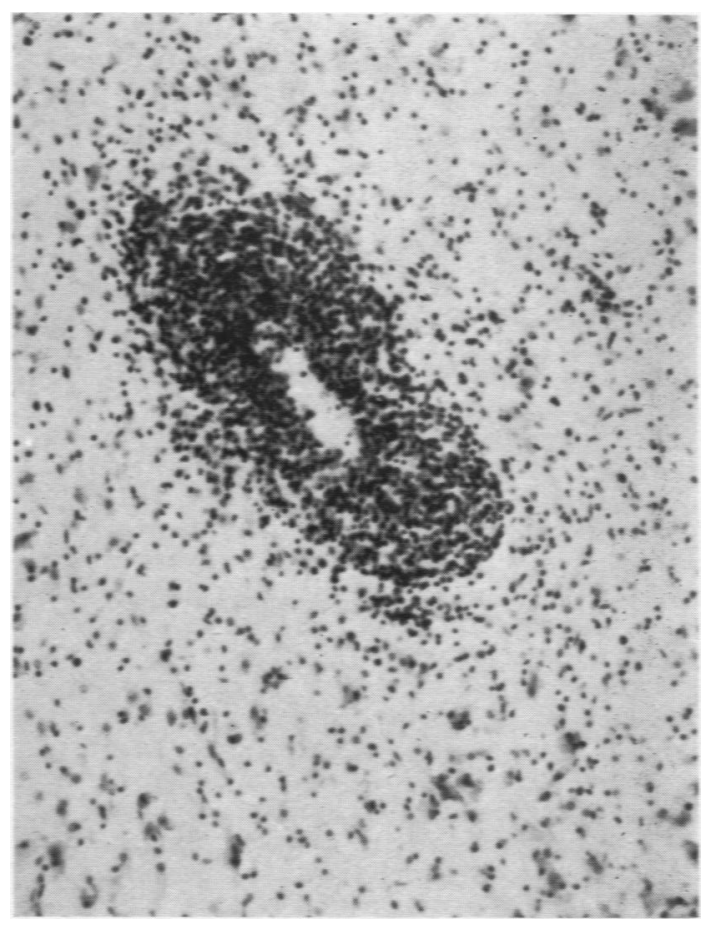

FrG. 2.-Thalamus, showing severe perivascular lymphocytic cuffing with activation of astrocytes around vessels and wandering of lymphocytes into tissues. Nissl $\times 112$.

showed activated astrocytes and proliferated microglia over a wider area. It was never possible to demonstrate definite infiltration or actual injury of the walls of vessels.

The right insula, both central regions and the dorsal convolutions of both frontal lobes showed extensive but patchy palings affecting the superficial layers or the 
whole thickness of the cortex (Fig. 4). Within these palings, the nerve cells showed a fine stippling of the chromatin of the nucleus, though sometimes larger globules of chromatin were present. The nucleolus was always enlarged and stained metachromatically. The cell body was sometimes of normal shape or slightly swollen, with an indistinctness or ground glass-like homogenization of the Nissl substance; at other times the cells were greatly swollen with a complete loss of Nissl substance and vacuole formation, recalling Nissl's 'water change'. Other cells showed more advanced degeneration, the nucleus being coarsely stippled and the cell body barely visible. Only very occasionally was encrustation of nerve cells seen. No marked change in the interstitial elements was observed. Of the various areas of cortex showing palings, that around the sulcus between the right middle and inferior frontal convolutions was the most severely affected.

The Sommer sector and endfolium of both Ammon's horns showed intense neuronophagia and ischaemic degeneration of nerve cells, with many rod cells and

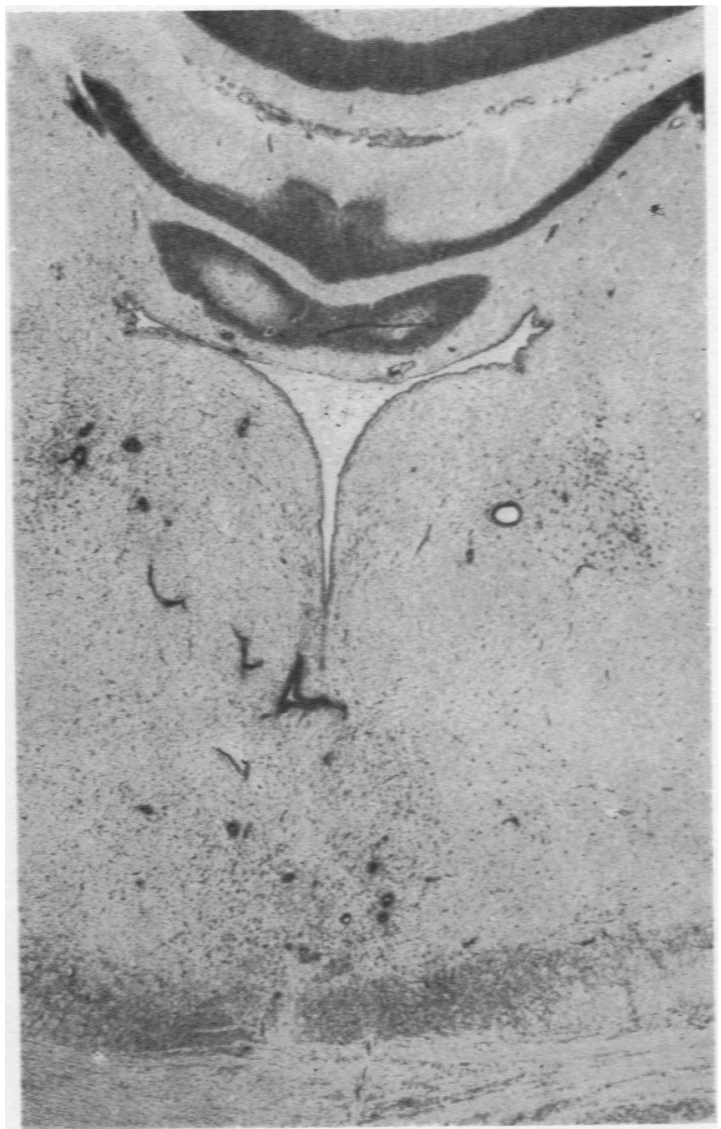

Fig. 3.-Upper part of pons, showing perivascular cuffing in periaqueductal grey matter. Nissl $\times 11$. amoeboid microglia and astrocytes showing Alzheimer's amoeboid change (Fig. 5). There was marked lymphocytic infiltration of the leptomeninges (Fig. 6).

\section{Discussion}

There appear to be in this case two distinct pathological processes: (1) A meningo-encephalitis characterized by severe lymphocytic infiltration of the subarachnoid space and thick perivascular cuffs of lymphocytes; some of the latter are infiltrating diffusely into the perivascular cerebral tissue, which shows a glial reaction suggestive of the effects of oedema. (2) Focal areas of selective parenchymal degeneration, considered to be typical of the effects of cerebral anoxia (Scholz, 1949), comprising the cortex of the right insula, both central regions and the dorsal convolutions of both frontal lobes, together with the endfolium and Sommer's

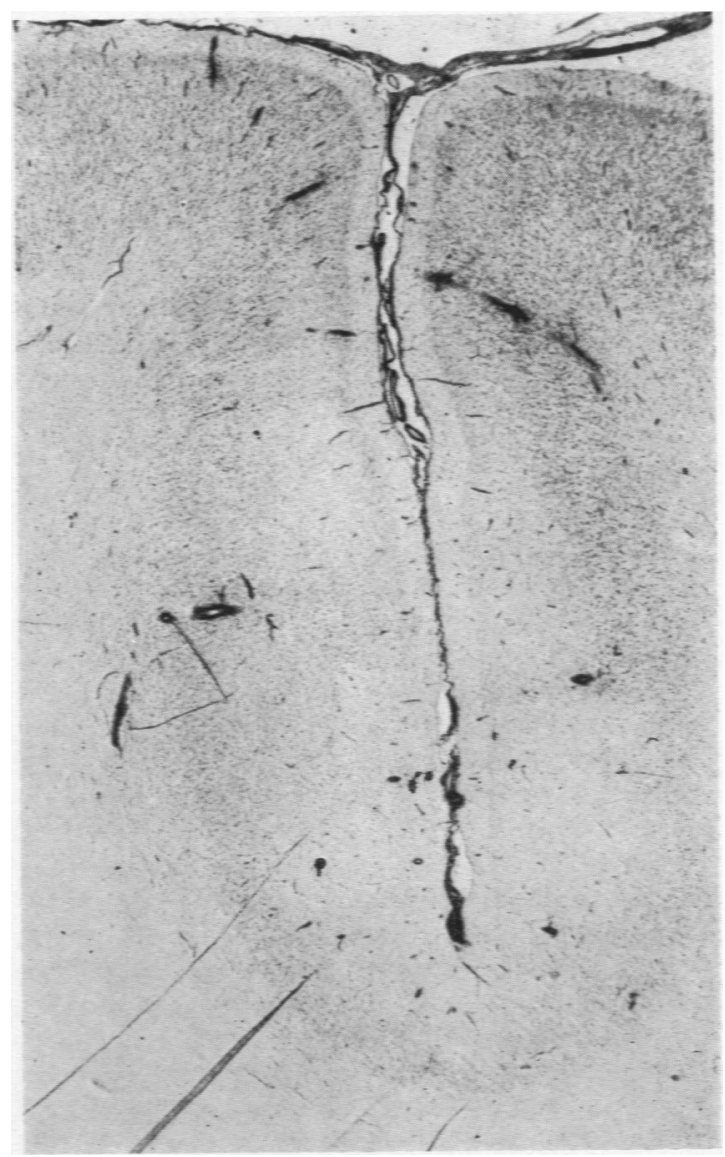

Fig. 4.-Right superior frontal convolution, showing paling of superficial layers of cortex, especially in depths of sukcus. Nissl $\times 40$. 


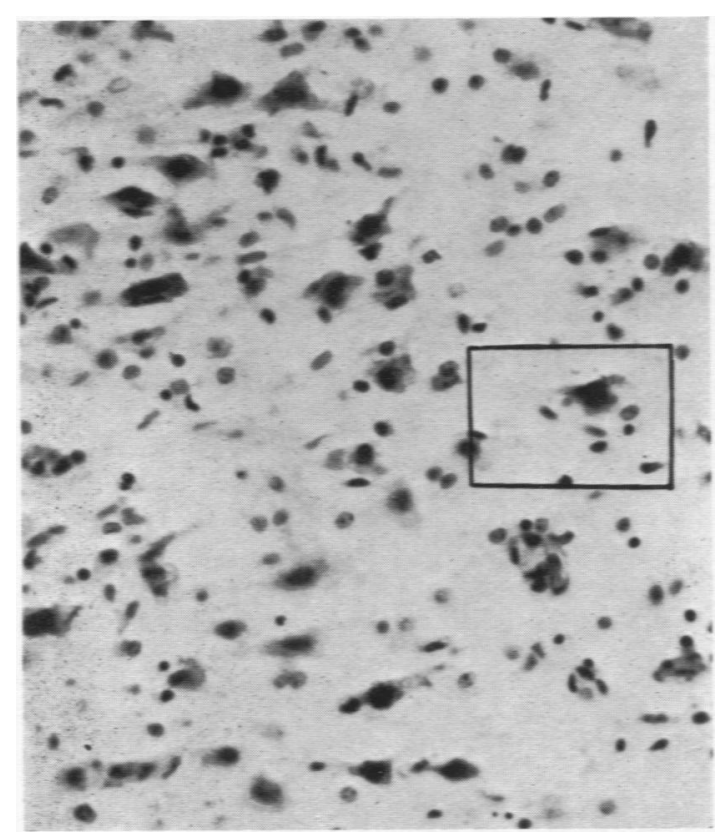

Frg. 5.-Ammon's horn, showing ischaemic nerve cells and microglial rod cells. The rectangle encloses an encrusted, ischaemic nerve cell.
Nissl $\times 300$.

sector of both Ammon's horns. The presence of many nerve cells showing acute ischaemic and homogenizing degeneration suggests that the anoxic episode responsible must have occurred within the last few days of life. While it is possible that the severe perivascular infiltrations may have caused minor circulatory disturbances, it seems unlikely that these were responsible for the sudden simultaneous development of widespread, very severe, focal anoxic lesions, confined to the cerebral cortex and Ammon's horn. On the other hand, just such a pattern of lesions is described by Scholz (1951) as an occasional finding in children dying in status epilepticus. Our patient was admitted in status epilepticus and the anoxic lesions are of an age consistent with having developed at this time. Their presence amply explains the depression of consciousness and focal neurological signs which followed the convulsions. It seems reasonable, therefore, to suggest that the anoxic lesions were caused in some way by the epileptic attack. If this view is accepted, it may be felt that some of the similar lesions seen in cases of 'whooping cough eclampsia' and regarded by Husler and Spatz (1924) as toxic and by Neubürger (1925) as the result of air embolism may in fact be the result of the convulsions. It may be significant that the neuro-

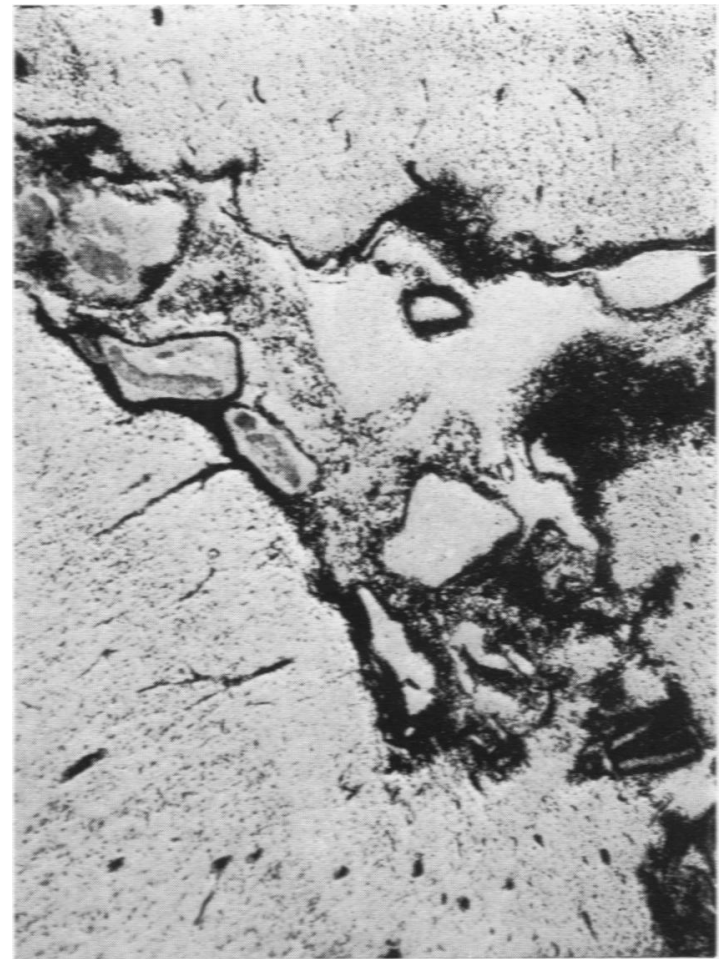

FK. 6.-Right pre-central region, showing heavy infiltration of the leptomeninges with lymphocytes. Nissl $\times 39$.

logical symptoms included convulsions in all reported cases of whooping cough eclampsia showing this type of selective parenchymal degeneration.

It remains to postulate a cause of the convulsions. In those cases in which fits supervene at the height of the whooping cough, a variety of causes may be put forward including toxaemia, air embolism and anoxia from respiratory embarrassment during a paroxysm. Indeed, as has recently been mentioned by Meyer, Beck and Shepherd (1955), severe epileptic convulsions may be precipitated by fevers, acute gastric infections and allergic crises. To this list, these authors add an important case in which status epilepticus complicated Still's disease. The patient survived the convulsions in a demented state for 12 months. By post-mortem examination the authors were able to demonstrate lesions which they consider to be associated with the original attack of status epilepticus. In this, they were guided by the topography and the histological character of the changes. Our case would probably have shown similar changes if survival had been equally prolonged. The cause of the convulsions in these 
cases often defies detection. Meyer and his collaborators were unable, in their case, to find any obvious nexus between the convulsions and any preceding event in the medical history. We are, perhaps, more fortunate. Examining the brain only two days after the onset of the convulsions, we were able to show meningo-encephalitic changes, such as might have been present in the case of Meyer $e t$ al. at a similar stage in its evolution. These changes may well have reflected the leucocytosis so well recognized in whooping cough (Seitz, 1925). It is remarkable that they have not been more frequently encountered in the brain. The 'encephalitic' changes in the case of Askin and Zimmerman (1929), much quoted in the American literature, included foci of mononuclear cellular infiltration and mononuclear and polymorphonuclear leucocytes in the sub-ependymal tissue of the lateral ventricle: nevertheless, the absence of perivascular cuffing or meningeal exudate prevents us from comparing the case too closely with our own. Habel and Lucchesi (1938) report early lymphocytic cuffing in the brain in one of seven cases subjected to necropsy. They state that it is evident that certain patients have 'definite' encephalitis as the underlying cause of the convulsions from the fact that convulsions occur when paroxysms are mild and bronchopneumonia is absent. They do not, however, give any examples or references to examples.

Considerable interest attaches to the report by Fonteyne and Dagnelie (1932) on the effects of intracerebral injection of $H$. pertussis toxin in guinea-pigs. The changes included (1) a very severe leucocytic meningeal reaction with predominance of polymorphonuclear leucocytes, (2) congestion of vessels, with numerous large perivascular cuffs of polymorphonuclear leucocytes and small lymphocytes in the cerebral peduncles and basal ganglia. It seems possible that in our case also the encephalitic changes resulted from the action of $H$. pertussis toxin. An alternative possibility is that the encephalitis was caused by some unknown and unrelated virus to which the whooping cough may or may not have lowered the resistance of the patient. In any case, the fact remains that a true encephalitis may occasionally occur during convalescence from whooping cough and cause convulsions in the absence of paroxysms of coughing; these convulsions, if sufficiently severe or accompanied by a sufficient degree of respiratory embarrassment, may cause anoxic degeneration of nerve cells especially in the cerebral cortex and Ammon's horn, with severe depression of consciousness, which may be immediately or rapidly fatal or lead to the permanent neuropsychiatric sequels (dementia, hemiplegia or generalized rigidity) well recognized (Ford, 1952) as following pertussis encephalopathy.

\section{Summary}

A case is reported in which convulsions fatal in 36 hours developed during convalescence from whooping cough at a time when there were no paroxysms of coughing.

Pathological examination of the brain showed very severe lymphocytic infiltration of the meninges and perivascular spaces constituting a meningo-encephalitis, together with degenerative lesions of an anoxic type, in scattered areas of cerebral cortex and in the Ammon's horn.

It is suggested that the meningo-encephalitis was responsible for the convulsions and that the anoxic changes were the result and not the cause of the fits. This contrasts with the situation in cases of whooping cough eclampsia where fits follow a paroxysm of coughing. In these cases anoxia, from air embolism or cerebral venous stasis, is usually considered to be the cause both of the convulsions and the nerve cell changes.

It is a pleasure to record our indebtedness to Professor Alfred Meyer for helpful advice during the investigation of this case.

\section{REFERENCES}

Askin. J. A. and Zimmerman. H. M. (1929). Amer. J. Dis. Child.. 38. 97.

Ford, F. R (1952). Diseases of the Nerrous System in Infancy. Childhood and Adolescence. 3rd ed., p. 693. Oxford.

Fonteyne, P. and Dagnelie, J. (1932). C. R. Soc. Biol. (Paris), 110,978 .

Habel, K. and Luochesi, P. F. (1938). Amer. J. Dis. Child., 56, 275.

Hiller, F. and Grinker, R. R. (1930). Arch. Neurol. Psychiat. (Chicago), 23, 634.

Husler, J. and Spatz, H. (1924). Z. Kinderheilk., 38. 428.

Neubürger, K. (1925). Klin. Wschr., 4, 113.

Meyer, A.. Bock, E. and Shepherd, M. (1955). J. Neurol. Neurosurg. Psychiat., 18, 24.

Scholz. W. (1949). Arch. Psychiat. Nervenkr., 181, 621. (1951). Die Krampfschädigungen des Gehirns. Monogr. Neurol. Psychiat., Heft 75. Berlin.

Seitz. R. P. (1925). Amer. I. Dis. Child., 30. 670. 